\title{
Indicateurs lexicaux dans les textes de comparaison et de problème-solution produits par des élèves de 8-9 ans
}

Renée Gagnon et Hélène Ziarko

\author{
(2) OpenEdition \\ Journals \\ Édition électronique \\ URL : http://journals.openedition.org/pratiques/3475 \\ DOI : 10.4000/pratiques.3475 \\ ISSN : 2425-2042 \\ Éditeur \\ Centre de recherche sur les médiations (CREM)
}

Édition imprimée

Date de publication : 15 décembre 2012

Pagination : $97-107$

Référence électronique

Renée Gagnon et Hélène Ziarko, «Indicateurs lexicaux dans les textes de comparaison et de problème-solution produits par des élèves de 8-9 ans », Pratiques [En ligne], 155-156 | 2012, mis en ligne le 18 décembre 2017, consulté le 10 décembre 2020. URL : http://journals.openedition.org/ pratiques/3475; DOI : https://doi.org/10.4000/pratiques.3475 


\title{
Indicateurs lexicaux dans les textes de comparaison et de problème-solution produits par des élèves de 8-9 ans
}

\section{Renée Gagnon}

\author{
Université du Québec à Trois-Rivières, Québec, Canada \\ Hélène Ziarko
}

Université Laval, Québec, Québec, Canada

\section{Introduction}

Les unités lexicales d'une production écrite constituent des indicateurs observables pour appréhender les défis linguistiques et cognitifs posés au scripteur. D'une part, elles mettent en évidence la disponibilité de ses ressources lexicales et son habileté à les mobiliser pour rendre « dicibles » (Dolz, Noverraz et Schneuwly, 2001) les contenus à développer en tenant compte des caractéristiques du genre de texte à produire. D'autre part, l'occurrence de certaines unités lexicales informe sur leur contribution au développement des processus cognitifs sous-jacents à l'établissement des différentes relations mises en cause dans le texte.

De par sa visée épistémique, le texte informatif sollicite particulièrement le recours à un lexique spécifique propre à soutenir tant le lecteur que le scripteur, dans le traitement des connaissances mobilisées dans le texte. La mise en mots est complexe puisque le scripteur doit à la fois linéariser le contenu sémantique dans la phrase et assurer la progression de l'information d'une phrase à l'autre selon l'organisation retenue (Gagnon et Ziarko, 2011). Ainsi, la mise en mots dépend des connaissances du scripteur, de l'étendue et de la justesse de son lexique ainsi que de sa capacité à l'utiliser judicieusement pour soutenir la construction des connaissances chez le lecteur.

Même si l'on reconnaît l'importance de l'enseignement-apprentissage de la lecture et de l'écriture du texte informatif, on observe qu'il occupe encore peu de place au début de l'école primaire, cela au profit du texte narratif, soit qu'on lui accorde peu de temps d'enseignement (Duke, 2000) ou soit qu'il est peu présent dans les ma- 
nuels de lecture (Moss et Newton, 2002). Pourtant, Hall, Sabey et Mc Clellan (2005) soulignent que négliger l'enseignement précoce du texte informatif pourrait contribuer à l'absence de progrès, voire au déclin, de l'efficacité en lecture des élèves après la $3^{\mathrm{e}}$ année ${ }^{(1)}$. Différentes causes possibles de cette régression ont été mises en évidence. Par exemple, nous savons que les jeunes enfants sont davantage exposés à la lecture et à l'écriture de récits, ce qui leur permet d'explorer davantage l'organisation canonique du texte narratif commune à tous les types de récits plutôt que les différentes structures du texte informatif qui, elles, varient selon la visée informative mise en évidence dans le texte (Sanacore et Palumbo, 2009). De plus, le peu d'occasions auxquelles les jeunes élèves sont confrontés à ce type de texte et à son lexique spécialisé ne favorisent pas le développement des habiletés nécessaires à sa compréhension et sa production, les textes informatifs étant pourtant de plus en plus utilisés dans l'enseignement des autres disciplines scolaires.

Depuis maintenant plusieurs années, nous avons étudié le rôle de différents facteurs sur l'enseignement-apprentissage de l'écriture du texte informatif par des élèves de $2^{\mathrm{e}}$ année et de $3^{\mathrm{e}}$ année, soit au début du primaire au Québec. Ces travaux ont d'abord concerné l'écriture du texte de comparaison (Gagnon, 2006 ; Gagnon et Ziarko, 2008 ; Gagnon et Ziarko, 2009) pour ensuite envisager celle du texte problème-solution (Gagnon et Ziarko, 2011 ; Gagnon, en cours). Au-delà des résultats montrant l'effet d'un enseignement spécifique de l'écriture de tels textes lié à certaines de leurs caractéristiques structurelles, nous voulons ici examiner les productions de nos élèves du point de vue des indicateurs lexicaux qui caractérisent ces deux types de textes, voire qui les particularisent.

\section{Le traitement des textes informatifs}

Depuis une trentaine d'année, les travaux menés par Meyer et ses collaborateurs (Meyer, 1985 ; Meyer et Poon, 2001 ; Meyer, Wijekumar, Middlemiss, Higley, Lei, Meier et Spielvogel, 2010) ont mis en évidence, d'abord, les différentes relations mises en œuvre dans les textes informatifs. Selon ces travaux, on trouve ainsi la description qui présente les caractéristiques d'un ou de plusieurs éléments ; la séquence qui présente les éléments en cause en suivant leur ordre de présentation temporelle ; la comparaison qui met en évidence les différences et les ressemblances entre deux ou plusieurs éléments; la relation de cause-effet qui explique les relations entre une cause et une conséquence; et celle correspondant à l'énoncé d'un problème et de sa solution qui permet de proposer une (des) solution(s) à un problème posé.

\subsection{La structure des textes informatifs}

Diverses études ont montré que ces relations rhétoriques ne sont pas équivalentes du point de vue du traitement exigé par le lecteur et le scripteur. Ce traitement concerne, d'une part, l'organisation des informations et le fait que celle-ci rende visible la prégnance de la relation qui correspond à la visée du texte et, d'autre part, les procédés linguistiques mis en œuvre dans l'écriture du texte. Ainsi, nous savons que les textes de comparaison et de problème-solution sont « les plus complexes, utiles et

(1) La $3^{\mathrm{e}}$ année du primaire (qui en compte 6 au Québec) correspond au cours élémentaire $2^{\mathrm{e}}$ année (CE2) en France. 
mémorisables » (Meyer et al., 2010, p. 63) du fait des enjeux de traitements cognitif et linguistique pour le lecteur. Dans le texte de comparaison, du fait de l'alternance des référents présentés, le lecteur doit effectuer des ruptures au cours du traitement des différents paragraphes, tandis qu'en même temps, il doit établir un lien de cohérence avec ce qui a été précédemment lu. Le scripteur, quant à lui, doit examiner les attributs qui caractérisent deux référents de façon à ne retenir que ceux sur lesquels il sera possible de se prononcer en termes de ressemblances et de différences. De plus, il peut recourir, au début ou à l'intérieur des paragraphes, à des connecteurs du type comme, par contre ou quant à pour renforcer la structure du texte et guider explicitement le traitement comparatif du lecteur.

Par ailleurs, Meyer (2003) a observé que la lecture de textes organisés selon une structure problème-solution causait des difficultés de compréhension aux élèves du primaire. Ainsi, une recherche qu'elle a menée auprès de lecteurs de $5^{\mathrm{e}}$ année a montré que la plupart d'entre eux ne saisissent pas la solution proposée pour résoudre le problème posé dans le texte. De plus, $70 \%$ de ces élèves n'ont pas été en mesure d'utiliser la structure problème-solution pour rendre compte du contenu d'un article de journal. La compréhension de ce type de texte exige, de la part du lecteur, l'établissement d'une relation logique de cause à effet entre des événements, relation qui est plus tardivement maîtrisée dans le développement cognitif de l'enfant. Quant au scripteur, il doit constituer l'événement problème en identifiant les faits mis en cause et expliquer les relations de causalité dans l'énoncé du problème et de la solution (Gagnon et Ziarko, 2011). Du point de vue du lexique, l'utilisation, par le scripteur, de connecteurs du type parce que, alors ou par conséquent et de verbes causatifs-résultatifs est nécessaire pour signaler explicitement au lecteur ces relations de causalité.

\subsection{Le traitement sémantique des textes informatifs}

La compréhension de la lecture d'un texte exige de la part du lecteur la mise en œuvre de processus cognitifs et l'utilisation des connaissances dont il dispose pour construire une représentation sémantique similaire à celle que l'auteur avait élaborée (Le Ny, 2005). Les processus d'identification de mots, d'activation de connaissances et d'instanciation de schémas, temporels ou causaux stockés dans la mémoire à long terme permettent au lecteur de construire un pont entre l'information textuelle et les connaissances du lecteur. Ainsi, le sens du texte se construit progressivement par l'apport successif d'informations nouvelles à des représentations précédemment activées ou encore à de l'information déjà énoncée et traitée.

Le lecteur construit une représentation de ce qui est dit par les mots et les phrases du texte en procédant à une série d'opérations de prédication (Le Ny, 1979) organisées en propositions sémantiques dont on peut établir la liste par l'analyse prédicative du texte (Denhière, 1983). Les propositions sémantiques sont constituées d'un prédicat, concept qui exprime une relation et d'un ou plusieurs arguments, objets de la relation qui peuvent être considérés comme «(des) concepts d'individu et aussi de catégorie » (Denhière et Beaudet, 1992, p. 51). Ainsi, le défi du scripteur consiste à linéariser ce qui est d'abord une représentation sémantique «globale » de façon telle que le lecteur puisse effectuer l'organisation de ces propositions à travers le texte, cela en assurant une progression sémantique cohérente dans la mise en relation (prédicats) des arguments.

La linéarisation du contenu sémantique du texte de comparaison mobilise essen- 
tiellement le lexique associé aux connaissances catégorielles ou relatives à l'organisation hiérarchique du monde. Elle vise la mise en relief de propriétés conduisant à établir des ressemblances et des différences entre deux référents pour en bout de ligne conduire classifier ces objets. Du point de vue du lexique, l'écriture de ce texte exige que le scripteur recoure à des substantifs qui permettent de nommer les catégories dans lesquelles classer ces référents ou à des adjectifs qui en désignent les propriétés. On observe ainsi que le contenu du texte de comparaison comporte un nombre élevé d'arguments (ce dont on parle) correspondant à la diversité des caractéristiques classifiant les référents présentés (par exemple : castor $=$ animal $=$ rongeur = mammifère). Par ailleurs, ce type de texte peut aussi inclure des mots propres à signaler la relation de comparaison, ces connecteurs appartenant aussi au lexique du scripteur.

Le texte problème-solution présente un événement problématique et l'action entreprise pour résoudre ce problème, la solution. Le défi du scripteur consiste, d'une part, à linéariser l'énoncé du problème en recourant à des informations (arguments) permettant de caractériser l'événement et de le constituer en problème. D'autre part, l'énoncé de la solution requiert qu'il procède à une reprise des arguments utilisés pour établir le problème et rendre explicites les relations de causalité (prédicats) reliant le problème à la solution proposée.

La linéarisation du contenu sémantique exige que le scripteur utilise des verbes ou des nominalisations (par exemple : utilisation pour utiliser) pour exprimer des actions reliées aux éléments mis en cause dans le problème et la solution. Par exemple, dans les phrases suivantes tirées d'un texte problème-solution :

\section{Problème: En effet, le faucon pèlerin avait presque disparu parce que l'utilisa- tion du pesticide DDT avait dévasté sa population. \\ Solution: Au Canada, on a décidé d'interdire l'usage de pesticide DDT.}

l'argument pesticide $D D T$ est présent dans l'énoncé du problème et repris dans l'énoncé de la solution tandis que la nominalisation utilisation correspond à l'action exprimée par ce prédicat mis en relation avec l'argument pesticide DDT.

Par ailleurs, afin de guider le lecteur dans l'établissement de ces relations, le scripteur doit utiliser des marqueurs du type parce que, alors, par conséquent pour les rendre explicites.

On observe que, à nombre de mots égaux, les analyses prédicatives des textes problème-solution présentent un nombre d'arguments moins élevé que celui observé dans les textes de comparaison, puisque certains arguments utilisés dans l'énoncé du problème sont repris dans l'énoncé de la solution associés à de nouveaux prédicats.

Dans le texte de comparaison, le nombre et la diversité des arguments révélés par l'analyse prédicative illustre une prise en charge paradigmatique se traduisant dans les choix lexicaux qui portent le contenu du texte. Par contre, les caractéristiques du texte de problème-solution axé sur la reprise d'arguments et sur l'explicitation de relations de cause-effet relèvent davantage de la mise en forme syntagmatique du discours, par ailleurs marquée par l'utilisation d'un lexique propre aux relations de causalité essentiellement destinées à soutenir la linéarisation du contenu de ce type de texte.

Ainsi, dans le but de vérifier si de telles différences entre ces deux types de textes pouvaient être observées dans ceux produits par des jeunes élèves au début du primaire, nous avons examiné un corpus de textes écrits par des élèves de 8 et 9 ans. 


\section{Méthodologie}

\subsection{Le corpus de textes}

Le corpus que nous avons examiné est issu de nos travaux antérieurs et comporte douze textes : 6 textes de comparaison dans lesquels les élèves devaient comparer 2 animaux par exemple, le loup gris et l'ours polaire et 6 textes de problème-solution dans lesquels les élèves devaient expliquer pourquoi le faucon pèlerin est en voie de disparition et quelles sont les solutions trouvées par les chercheurs pour résoudre ce problème. Les textes comparés comptaient sensiblement le même nombre de mots et n'avaient pas fait l'objet d'une correction par les élèves.

Globalement, les activités d'enseignement auxquelles avaient participé les élèves avant l'écriture du texte étaient relativement semblables. Ainsi, en $2^{\mathrm{e}}$ année, ces activités ont porté sur la lecture de textes de comparaison, l'enseignement spécifique de la structure du texte, caractérisée par l'alternance des paragraphes dans lequel est présenté chaque aspect de chacun des référents décrits, et l'enseignement des marqueurs de comparaison. En $3^{\mathrm{e}}$ année, les activités ont été axées sur la lecture de textes problème-solution, l'enseignement de la structure du texte caractérisée par la segmentation en quatre parties (introduction, problème, solution, conclusion), la reprise de certains arguments du problème dans la solution et l'enseignement des marqueurs de causalité.

\subsection{L'instrument de mesure}

Dans le but de mettre en évidence les capacités de jeunes scripteurs, qui commencent à peine l'apprentissage de l'écriture, à linéariser un contenu sémantique, nous avons procédé à l'analyse prédicative de textes d'élèves de $2^{\mathrm{e}}$ et $3^{\mathrm{e}}$ année ${ }^{(2)}$ produits dans le cadre des travaux précédemment mentionnés. Cette analyse est reprise des travaux de Le Ny (1979) et de Denhière (1983) et elle s'apparente à celle rapportée par Kintsch et Van Dijk (1978) ; ces analyses ont été mises au point pour rendre compte du fonctionnement de la mémoire sémantique. L'analyse prédicative permet d'identifier les différentes propositions sémantiques contenues dans les textes. Chaque proposition correspond à une relation exprimée par un prédicat mettant en cause un ou n arguments (Denhière, 1983) et toutes les propositions sémantiques d'un texte sont interreliées afin de former un ensemble cohérent compréhensible par le destinataire. Ces prédications permettent au lecteur de construire pas à pas la représentation mentale de ce qui est dit par le texte (Denhière et Beaudet, 1992). Ainsi, un texte est construit comme une suite de propositions sémantiques, chacune d'elles constituant une unité d'information formée d'au moins deux concepts dont l'un joue le rôle d'argument (l'ensemble des arguments étant représenté par des variables x1, $\mathrm{x} 2$, etc.) tandis que l'autre joue le rôle de prédicat. Par ailleurs, une proposition sémantique peut devenir l'argument d'une proposition subséquente.

L'analyse prédicative permet de comparer entre eux des textes au niveau de la microstructure sémantique en observant le nombre de propositions sémantiques et d'arguments qu'ils contiennent. Elle permet également de rendre compte de la densité sémantique des textes.

(2) La 2e année du primaire du Québec correspond à la $1^{\text {re }}$ année du cours élémentaire CE1 en France (cycle 2, 7-8 ans). 
Selon Kintsch et Van Dijk (1978), la densité sémantique d'un texte correspond au rapport entre le nombre de propositions sémantiques et le nombre d'arguments contenus dans un texte.

Par exemple (Tableau 1), comparons deux extraits de textes comportant sensiblement le même nombre de mots, pour l'un tiré d'un texte de comparaison $(\mathrm{N}=26)$, et pour l'autre d'un texte problème-solution $(\mathrm{N}=24)$.

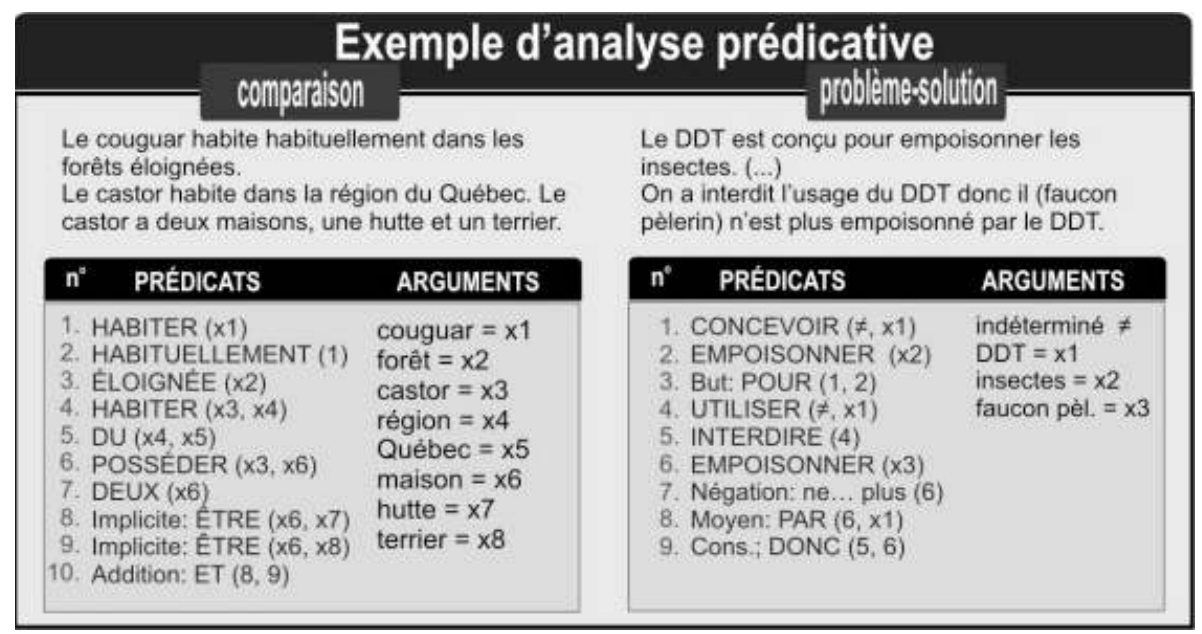

Tableau 1: Exemple d'analyse prédicative

On peut observer que la densité sémantique est moins élevée dans l'extrait du texte de comparaison (10 propositions sémantiques pour 8 arguments ou une densité sémantique de 1,25) que dans l'extrait du texte problème-solution ( 9 propositions sémantiques pour 4 arguments ou une densité sémantique de 2,25).

Dans l'extrait du texte de comparaison, les arguments correspondent aux propriétés $(\mathrm{x} 2, \mathrm{x} 4, \mathrm{x} 5, \mathrm{x} 6, \mathrm{x} 7, \mathrm{x} 8)$ permettant de comparer les deux référents, le couguar et le castor (x1, x3). Par ailleurs, un même prédicat (HABITER) relie ces arguments. Dans l'extrait du texte de problème-solution, les arguments indéterminé = on et DDT appartiennent à l'énoncé du problème ( $1^{\text {re }}$ phrase) et sont repris dans la solution $\left(2^{\mathrm{e}}\right.$ phrase $)$, reliés par des prédicats différents, en particulier un but posant problème (proposition 3) et un moyen (proposition 8) dont la conséquence (proposition 9) correspond à la solution proposée. Nous avons ici un nombre réduit d'arguments inscrits dans un nombre plus élevé de propositions. Toutefois, la densité sémantique plus grande du deuxième extrait (texte de problème-solution) peut être un élément augmentant la complexité du traitement, dans la mesure où ce nombre restreint d'arguments fait l'objet de relations logiques établissant d'abord le point de vue du problème et ensuite celui de la solution. Nous savons que le traitement de telles relations est encore difficilement maîtrisé par des élèves au début du primaire. En ce sens, le traitement exigé par la lecture et l'écriture du texte de problème-solution et du texte de comparaison ne mobilise pas de la même façon les ressources lexicales des élèves. L'écriture du texte de comparaison fait surtout appel à la diversité des connaissances lexicales permettant de nommer les propriétés des référents (arguments) à catégoriser tandis que l'écriture du texte de problème-solution ne peut se dispenser de mobiliser les indicateurs lexicaux (prédicats) nécessaires à l'établissement de relations logiques explicites. 


\section{Résultats}

\subsection{La densité sémantique des textes produits}

Nous avons réalisé l'analyse prédicative des douze textes du corpus qui nous a permis d'établir la densité sémantique des deux types de textes. On calcule la densité sémantique d'un texte en divisant le nombre de propositions sémantiques (prédicats) qu'il contient par celui du nombre d'arguments. Les résultats montrent que, avec un nombre sensiblement égal de mots, les densités sémantiques (ou ratios prédicats-arguments) sont moins élevées dans les textes de comparaison (de 1,57 à 2,33) que dans les textes de problème-solution (de 2,57 à 4,18).

\begin{tabular}{|c|c|c|c|c|c|c|c|}
\hline \multicolumn{8}{|c|}{$\begin{array}{l}\text { DENSITÉ SÉMANTIQUE DES TEXTES } \\
\text { nparaison } \\
\text { problème-solution }\end{array}$} \\
\hline$n$ mots & pred & arg & ratio & $\mathrm{n}$ mots & pred & arg & ratio \\
\hline 163 & 49 & 25 & 1,96 & 163 & 72 & 28 & 2,57 \\
\hline 160 & 64 & 33 & 1,93 & 158 & 48 & 15 & 3,2 \\
\hline 132 & 55 & 29 & 1,89 & 131 & 46 & 11 & 4,18 \\
\hline 116 & 49 & 21 & 2,33 & 116 & 47 & 13 & 3,61 \\
\hline 88 & 28 & 17 & 1,64 & 88 & 23 & 9 & 2,55 \\
\hline 78 & 30 & 19 & 1,57 & 78 & 32 & 10 & 3,2 \\
\hline
\end{tabular}

Tableau 2 : Densité sémantique dans les deux types de textes

En particulier, le troisième exemple du tableau 2 ci-dessus montre une différence notable entre les résultats. Ainsi, pour des textes qui comportent 131 et 132 mots, le texte de comparaison compte 29 arguments pour 55 propositions sémantiques soit une densité sémantique de 1,89. Le texte de problème-solution compte, quant à lui, 11 arguments pour 46 propositions sémantiques pour une densité sémantique de 4,18. Autrement dit, dans le cas du texte de comparaison, chacun des arguments entre en moyenne dans deux propositions sémantiques qui sont le plus souvent rattachées à l'un et à l'autre des deux référents comparés. Par contre, dans le texte de problème-solution, chaque argument est repris dans environ quatre propositions sémantiques et participe à l'établissement de quatre relations différentes. Ainsi, dans ce dernier cas, chaque argument se trouve au sein d'un faisceau de relations plus important ou plus dense distribuées entre l'énoncé du problème et celui de la solution (Figure 1). Par exemple, on peut trouver les deux propositions : 1. UTILISER (DDT) et 2. TROP (1) dans l'énoncé du problème, et dans l'énoncé de la solution la proposition : INTERDIRE (DDT).

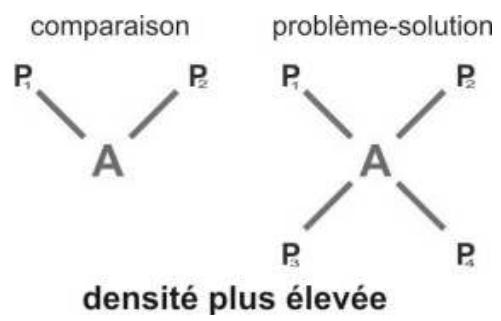

Figure 1 : Illustration des relations prédicats-arguments dans les deux types de textes 


\subsection{Les marqueurs de relation}

Du point de vue des marqueurs de relation, le tableau 3 montre que les scripteurs n'ont que peu ou pas utilisé de marqueurs pour signaler la relation de comparaison, ce qui n'invalide pas la comparaison effectivement réalisée puisque dans tous ces textes, elle a été soutenue par l'alternance des paragraphes.

\begin{tabular}{|c|c|c|c|}
\hline \multicolumn{4}{|c|}{$\begin{array}{c}\text { de } \\
\text { comparaison }\end{array}$} \\
\hline Texte & & cause & conséquence \\
\hline 1 & - & parce que, grâce à (i) & par conséquent, donc, donc \\
\hline 2 & par contre & parce que, grâce à & alors, alors, le fait de... c'est que \\
\hline 3 & - & à cause de, grâce à (i) & alors \\
\hline 4 & alors & à cause & par conséquent, donc, ainsi \\
\hline 5 & - & à cause & alors, donc \\
\hline 6 & - & - & ainsi, bien, (alors) \\
\hline
\end{tabular}

Tableau 3 : Marqueurs de relation relevés dans les textes de comparaison et de problème-solution

En effet, dans le texte de comparaison, le scripteur peut juxtaposer les informations concernant alternativement l'un ou l'autre des référents sans établir de lien explicite entre ces informations (texte A en annexe). Par contre, dans les textes de problème-solution (texte $\mathrm{B}$ en annexe), les élèves ont eu recours à des marqueurs logiques pour signaler explicitement aux lecteurs la relation de causalité entre les éléments du problème et les solutions proposées. Par ailleurs, nous avons aussi observé que, dans certains textes, la relation de causalité demeure implicite comme dans la phrase suivante tirée du texte 3 : «L'élevage en captivité a permis au faucon pèlerin de se reproduire. " où la relation deviendrait explicite par l'ajout d'un marqueur comme « grâce à »; cette situation est indiquée dans le tableau par l'indice (i).

\subsection{Verbes « d'action » ou de « changement»}

Concernant précisément les textes de problème-solution, nous avons souligné précédemment l'importance des prédicats dans l'établissement du problème et de la solution proposée. En lien avec cette constatation, nous avons comparé les caractéristiques sémantiques des verbes (prédicats) utilisés par les scripteurs dans les deux types de textes pour exprimer les relations établissant le problème, d'une part, la solution, d'autre part. Pour ce faire, nous avons relevé la présence de verbes dits «causatifs » parce que signalant la cause d'un effet favorable ou non et les verbes dits « résultatifs » indiquant la conséquence ou l'effet produit (Le Ny, 2005). Par exemple, dans l'analyse prédicative de l'extrait du texte de problème-solution (Tableau 1), le prédicat EMPOISONNER (insecte $=\mathrm{x} 2$ ) serait un verbe causatif dont on n'indique pas explicitement le résultat. Par contre, INTERDIRE (DDT) c'est causer un changement (verbe causatif) par lequel une action qui était possible devient impossible, EMPOISONNER (faucon pèlerin $=x 3$ ). Dans les six textes de problème-solution du corpus, nous avons pu observer que les scripteurs ont eu recours à ces deux types de verbes pour expliquer le problème et/ou la solution. De plus, fréquemment (dans 4 textes 
sur 6), un couplage de verbes causatifs-résultatifs a été opéré dont le plus fréquent est «manger/empoisonner ». Nous avons également relevé les couplages suivants : empoisonner/tuer; empoisonner/la coquille être fine ; mettre en captivité/sauver; se nourrir/empoisonner ; se reproduire/avoir beaucoup augmenté ; interdire/ne plus empoisonner ; menacer/avoir de moins en moins (de faucons pèlerins) ; interdire/avoir de plus en plus (de faucons pèlerins). Certains de ces couplages permettent d'énoncer le problème : empoisonner/tuer, empoisonner/la coquille être fine, se nourrir/empoisonner, menacer/avoir de moins en moins; d'autres énoncent la solution : interdire/ne plus empoisonner, mettre en captivité/sauver, interdire/avoir de plus en plus.

\section{Conclusion}

L'examen des textes du corpus auquel nous avons procédé révèle une certaine sensibilité des élèves concernant le lexique et plus précisément le choix des mots qui permettent de linéariser le contenu d'un texte informatif. De plus, nos observations montrent aussi leur capacité d'utiliser le lexique de façon différenciée selon le type de texte informatif à produire. Ainsi, l'analyse prédicative des textes de comparaison met en évidence l'étendue du lexique permettant d'opérer les choix paradigmatiques nécessaires à l'énoncé des propriétés (le plus souvent des noms) responsables de la catégorisation des référents, objets de la comparaison. Dans ces textes, les relations exprimées par les propositions sémantiques sont essentiellement destinées à établir un lien entre chacun des référents et une propriété, c'est-à-dire entre des arguments. On observe souvent que le verbe ou l'adjectif permettant d'établir ce lien (prédicat) est répété pour l'un et l'autre des référents. De plus, à l'occasion, mais encore peu souvent, l'exploitation du lexique se traduit aussi par l'ajout d'un ou de plusieurs marqueurs de relation soulignant explicitement la comparaison déjà établie par l'alternance des paragraphes qui décrivent chacun des référents concernés.

L'analyse prédicative des textes de problème-solution révèle que les arguments nécessaires pour nommer les éléments du problème et de la solution sont comparativement moins nombreux pour plus de propositions sémantiques que ce qui est observé dans les textes de comparaison de même longueur, ceci conduisant à une plus grande densité sémantique pour les premiers. On a pu observer qu'un même argument pouvait entrer dans plusieurs propositions sémantiques pour expliquer le problème, d'une part, et qu'il pouvait être repris dans plusieurs autres propositions sémantiques pour expliquer la solution.

Ainsi, dès la $3^{\mathrm{e}}$ année, l'enseignement de l'écriture d'un tel texte a fait en sorte de rendre les élèves capables d'utiliser les unités lexicales pertinentes exigées par l'établissement de la cohérence du texte. Par ailleurs, la cohérence des textes est aussi assurée par une utilisation appropriée de couples de verbes causatifs/résultatifs dans la mise en relation de ces arguments, ce couplage de verbes contribuant à établir l'adéquation de la solution par rapport au problème posé. Enfin, l'analyse a aussi montré que, à la différence des textes de comparaison, les textes de problème-solution comportent une diversité de marqueurs de relation sans lesquels les relations logiques de causalité demeureraient implicites, voire non établies. Il ressort de notre analyse que les élèves de $3^{\mathrm{e}}$ année commencent à mettre en œuvre les procédés linguistiques susceptibles d'assurer la continuité syntagmatique de l'écriture d'un texte de problèmesolution. En particulier, ils sont en mesure d'utiliser la reprise pertinente d'arguments, de mobiliser des verbes causatifs-résultatifs en guise de prédicats et de recourir à des indicateurs lexicaux propres à assurer la mise en relation logique du problème et de sa solution. 
Ces résultats, certes fondés sur un échantillon restreint de productions, nous permettent de penser qu'un enseignement de l'écriture de textes informatifs de comparaison et de problème-solution est non seulement possible mais nécessaire, cela dès le début du primaire. Cet enseignement doit viser tant l'apprentissage des caractéristiques structurelles que celui des indicateurs lexicaux qui distinguent ces deux types de textes.

\section{Bibliographie}

DENHIÈRE, G. (1983) : «Ouvrir (x, fenêtre) et ouvrir (x, yeux). De l'analyse expérimentale à l'étude sur le terrain de la compréhension de textes ». Rééducation orthophonique, 21, 431-451.

DENHIÈRE, G. et BEAUDET, S. (1992) : Lecture, compréhension de texte et science cognitive. Lille : Presses universitaires de Lille.

Dolz, J., NoverRaZ, M. et SCHNEUWLy, B. (2001) : S'exprimer en français : séquences didactiques pour l'oral et pour l'écrit. Bruxelles : De Boeck.

DUKE, N.K. (2000) : « 3.6 minutes per day: The Scarcity of Informational Texts in the First Grade ». Reading Research Quaterly, 35, 202-224.

GAGNON, R. (2006) : Les effets d'une séquence didactique sur les opérations de textualisation d'un texte documentaire de comparaison par des élèves de $2^{e}$ année $d u$ primaire. Thèse de doctorat inédite. Québec : Université Laval.

- (2009-2012): L'enseignement et l'apprentissage de l'écriture du texte de type problème-solution en $3^{e}$ année du primaire : interactions entre lecture, écriture et communication orale. Projet de recherche subventionné par le Fonds de recherche sur la société et la culture, Québec.

GAGNON, R. et ZiARKO, H. (2008) : L'apport de la lecture de textes sources dans l'écriture des textes documentaires par des élèves de $2^{\mathrm{e}}$ année du primaire. Revue canadienne de linguistique appliquée, 11 (2), 9-26.

- (2009) : Apprendre à écrire un texte documentaire de comparaison en $2^{\mathrm{e}}$ année du primaire : étude comparée d'interventions didactiques contrastées. Revue des sciences de l'éducation, 35 (3), 127-148.

- (2011) : «Le texte informatif : un outil langagier pour apprendre ». Québec Francais, 161, 50-53.

KinTSCH, W. et VAN DiJK, T. A.(1978) : « Toward a Model of Text Comprehension and Production ». Psychological Review, 85, 363-394.

HALl, M.K., S ABEY, L.B. et MC Clellan, M. (2005) : «Expository Text Comprehension : Helping Primary Grade Teachers Use Expository Texts to Full Advantage ». Reading Psychology. 26, 211-234.

LE NY, J-F. (1979) : La sémantique psychologique. Paris : PUF.

- (2005) : Comment l'esprit produit du sens. Paris : Odile Jacob.

MEYER, B.J.F. (1985) : « Prose Analysis : Purpose, Procedures and Problems ». In B.K. Britton et J.B. Black. (dir.), Understanding expository text (pp.11-64). Hillsdale, $\mathrm{NJ}:$ LEA.

— (2003): «Text coherence and readability ». Topics in Language Disorders, 23, 204-221.

MEYER, B. J. F. et PoON, L. W. (2001) : « Effects of Structure Strategy Training and Signaling on Recall of Text ». Journal of Educational Psychology. 93(1), 141-159.

Meyer, B. J. F. WijeKumar, K., Middlemiss, W., Higley, K., Lei, P-W., Meier, C., 
Spielvogel, J. (2010) : «Web-Based Tutoring of the Structure Strategy With or Without Elaborated Feedback or Choise for Fifth-and Seventh-Grade Readers ». Reading Research Quarterly, 45(1), 62-92.

Moss, B. et NEWTON, E. (2002) : «An Examination of the Informational Text Genre in Basal Readers ». Reading Psychology, 23, 1-13.

SANACORE, J. et PAlumbo, A. (2009) : " Understanding the Fourth-Grade-Slump : Our Point of View ». The Educational Forum, 73, 67-74.

\section{Annexe \\ Exemples de textes produits ${ }^{(3)}$}

Texte A

Le castor et l'ours polaire (élève de $2^{\mathrm{e}}$ année, 8 ans)

Le castor est herbivore. Par contre l'ours est carnivore.

Le castor a les dents jaunes. Sa queue est plate et sa queue a des écailles. Les bébés ont une fourrure douce.

L'ours polaire est énorme. Sa fourrure est épaisse et blanche. Il a une petite queue. Il a le museau pointu.

Le castor vit dans une hutte, il vit sous l'eau et sur la terre.

L'ours polaire vit à la toundra arctique au Pôle Nord, le long de l'océan Arctique.

Le castor mange des nénuphars, des racines, des brindilles, l'écorce des arbres et de l'herbe.

L'ours polaire mange du poisson, du phoque, (des) bœufs musqués, (des) fruits, (des) lièvres, (des) caribous et (des) feuilles d'arbustes.

Le castor se défend avec ses dents et des coups de pattes.

L'ours polaire se défend avec ses pattes, ses griffes et ses crocs.

Le castor et l'ours polaire ont été en danger de disparition. Mais maintenant on se force pour les protéger.

Texte B (élève de $3^{\mathrm{e}}$ année, 9 ans)

Le faucon pèlerin est en voie de disparition.

Le pesticide DDT est un produit qu'on met pour éloigner les insectes et ca les empoisonne et après ça les tue. Alors ca fait une chaine alimentaire : les insectes qui sont empoisonnés après ce sont les petits mammifères qui sont empoisonnés parce que ils se mangent et ca fait la chaine alimentaire et après c'est les faucons pèlerins qui mangent les petits mammifères alors les faucons pèlerins sont empoisonnés et quand ils vont faire des bébés la coquille du bébé va être fine.

Pour sauver les faucons pèlerins ils les ont mis en captivité et le fait de les avoir mis en captivité c'est que les hommes ou les femmes s'occupent d'eux les faucons pèlerins bien sûr et (l') autre solution c'est d'interdire le pesticide DDT.

Grâce à l'élevage en captivité et à l'interdiction du DDT le faucon pèlerin sa survie est moins menacée et aussi il reste un faucon pèlerin à Trois-Rivières.

(3) Ces exemples respectent les versions manuscrites produites par les élèves. Seules les orthographes d'usage et grammaticale ont été corrigées. 